\title{
ORIGINALIDAD SUBJETIVA Y COPYRIGHT. EL CASO DEL FLAMENCO EN ESPAÑA
}

\section{CREATIVE SUBSTANCE AND COPYRIGHT. THE CASE OF FLAMENCO IN SPAIN}

Jesús Heredia-Carroza Universidad de Sevilla jhercar12@gmail.com

Luis Palma Martos

Universidad de Sevilla Ipalma@us.es

Luis F. Aguado

Pontificia Universidad Javeriana de Cali Ifaguado@javerianacali.edu.co

Resumen: El artículo pone de manifiesto que los derechos de autor como mecanismo de remuneración y protección del trabajo creativo presentan una limitación en su cobertura. El papel del intérprete en el caso del flamenco en España ilustra esta afirmación. En el artículo a partir de elementos conceptuales de la Economía de la Cultura (valor cultural) y del Derecho Comparado (originalidad subjetiva) se construye un esquema metodológico que permite identificar elementos clave del valor cultural aportado por el intérprete a la obra, p.ej. la altura creativa, la variación distinguible y la novedad suficiente, que, a su vez, le generan un sello distintivo (originalidad subjetiva), que sustenta la demanda de una mayor cobertura vía derechos de autor.

Palabras clave: Flamenco; intérprete; copyright; valor cultural; originalidad subjetiva.

\begin{abstract}
The paper shows that copyright is limited as a mechanism for rewarding and protecting creative work. The role of flamenco performers in Spain illustrates this statement. Through conceptual elements of Cultural Economics (cultural value) and Comparative Law (creative substance), we designed a methodology that allows for identification of the key elements (e.g., the creative height, distinguishable variation and novelty) that the interpreter contributes to the cultural value of the work. These elements produce a mark that reflects the creative substance of the performer, and this mark supports the demand for greater protection via copyright.
\end{abstract}

Keywords: Flamenco; performer; copyright; cultural value; creative substance. 


\section{Introducción}

Es una realidad que la normativa jurídica (p.ej. la española) que regula los derechos de autor evidencia una mayor cobertura, protección jurídica y, por tanto, patrimonial para los autores frente a los intérpretes. ¿Cómo explicar esta realidad? Pareciera que subyace la idea de que el trabajo creativo de los autores (p.ej. compositores de letra y melodía) es superior, o merece mayor protección, frente al trabajo realizado por los intérpretes. O desde otro punto de vista, pareciera que el trabajo de los intérpretes puede caer en un tipo especial de trabajo rutinario, no creativo, de bajo valor cultural (Caves, 2003: 73) y de menor consideración en el marco de la protección jurídica que implica los derechos de autor. El caso de los cantaores, tocaores y bailaores flamencos ejemplifica lo anterior.

Haciendo referencia al Texto Refundido de la Ley de Propiedad Intelectual español ${ }^{1}$ (TRLPI, en adelante), se observa cómo la ubicación de los derechos del autor y del intérprete es diferente (de Román, 2010: 1). En el Libro I² se recogen los derechos de los autores, mientras que en el Libro II $^{3}$ aparecen además de los intérpretes, los productores de fonogramas, las entidades de radiodifusión, entre otros. De esta forma en el Libro II surgen titulares de diversa naturaleza; por un lado, los intérpretes que aportan entidad creativa a la obra flamenca (Levinson, 2015: 76) y, por otro, los que participan con recursos no creativos, como son los financieros o técnicos. De ello se desprende la idea de que el TRLPI español diferencia dos niveles de trabajo creativo, cada uno de ellos regulado por un libro diferente (autores e intérpretes).

En el Libro I estarían los conocidos como derechos de autor y en el Libro II los derechos vecinos. Como se verá más adelante, la diferencia en su cobertura, tanto moral como patrimonial, es sustancial, lo que acentúa la diferencia entre los trabajos creativos realizados por el autor y el intérprete. Otra de las diferencias es la duración de los derechos patrimoniales, ya que los derechos de los autores duran toda su vida y setenta años después de su muerte o declaración de fallecimiento (art. 26); no obstante, los de los artistas duran setenta años desde la interpretación o desde la divulgación lícita de la grabación de su ejecución (Directiva 2011/77/EU). Esto crea un desequilibrio que afecta a la última etapa de la vida de los intérpretes, que es cuando no pueden realizar su principal actividad (conciertos, galas, grabaciones, etc.) y más necesitan la protección y remuneración derivada de su trabajo creativo.

En el artículo, la metodología utilizada para argumentar la brecha existente entre la importancia del trabajo creativo del intérprete y su protección por parte de los derechos de propiedad intelectual, se sustenta en tres aproximaciones. La primera de ellas, está basada en elementos de Derecho Comparado, a fin de analizar las diversas opciones y controversias que se plantean desde el punto de vista jurídico.

1 Real Decreto Legislativo 1/1996, de 12 de abril, por el que se aprueba el texto refundido de la Ley de Propiedad Intelectual regularizando, aclarando y armonizando las disposiciones legales vigentes sobre la materia.

2 El Libro I se denomina "de los Derechos de autor" en el Real Decreto Legislativo 1/1996, de 12 de abril.

3 El Libro II aparece en el Real Decreto Legislativo 1/1996, de 12 de abril como "de los otros derechos de propiedad intelectual y de protección sui generis de las bases de datos, junto con otros titulares de derechos de propiedad intelectual. 
En segundo lugar, se plantea un modelo 4 que permite capturar tanto el valor cultural como el valor económico subyacente. Por último, se utilizan los elementos de los marcos metodológicos anteriores para explorar su utilidad a partir del análisis de dos casos.

El artículo constituye un aporte a la literatura de la Economía de la Cultura centrado en el análisis de la valoración del flamenco, pudiendo ser extrapolable a otros géneros musicales, donde la importancia del trabajo creativo del intérprete es notable. Más concretamente, se ha diseñado un esquema metodológico útil que refleja el aporte creativo del intérprete, la forma de identificarlo en términos del valor cultural y el desequilibrio generado por el instrumento diseñado para su protección: los derechos de autor.

\section{Marco conceptual: la originalidad subjetiva}

Para comenzar, se aborda el concepto de originalidad subjetiva. Para esta tarea, se usan diferentes regulaciones legales de Derecho Comparado, con el objetivo de dejar sentadas las bases desde una perspectiva jurídica transversal.

Las leyes francesas ${ }^{5}$, no tratan directamente el concepto de originalidad como criterio de protección, aunque sí lo ha utilizado la Cour de Cassation. Se menciona la originalidad subjetiva, que se observa en la impronta del autor en la obra, independientemente de si la obra es novedosa o no ${ }^{6}$.

La persönliche geistige Shöpfungen (Ley de Creaciones Intelectuales Personales Individuales alemana) en su artículo 2.2., incorpora el concepto de originalidad subjetiva (de Román, 2003: 39), tratando la doctrina y la jurisprudencia la incorporación de la altura creativa -Gestaltungshöhe- como requisito; dicho estándar de originalidad es el más exigente de Europa. Para considerar la originalidad de una obra en Alemania, es necesario un nivel mínimo de novedad, que puede ser fijado de forma distinta, dependiendo de la naturaleza de la creación y del grado de libertad creativa que tenga el autor. Siguiendo la línea marcada por Loewenheim (de Román, 2003: 172), la obra debe mostrar el "espíritu humano" -der menschliche Geist- y la individualidad de su creador". De esta manera el autor tiene libertad de elección en la concepción de la obra para dotarla de su impronta o sello personal.

4 Para el diseño del modelo, se realizaron 15 entrevistas en profundidad a expertos en flamenco y de la cultura en general que incluyen, entre otros a intérpretes, autores, gestores de espacios culturales o representantes de las sociedades de gestión de derechos. El criterio de selección de los entrevistados fue su relevancia en el mundo del flamenco (p.ej. artistas flamencos que hayan obtenido reputados premios nacionales e internacionales, gestores que dirijan importantes festivales y espacios culturales -p.ej. Bienal de Flamenco de Sevilla-). En el Anexo 1 se presenta el listado respectivo y la característica distintiva de cada entrevistado.

5 Loi relative aux droits d'auteur et aux droits des artistes-interprètes, des producteurs de phonogrammes et de vidéogrammes et des entreprises de communication audiovisuelle. Collection de lois accessible en ligne, $\mathrm{n}^{\circ}$ 85-660, du 3 juillet 1985. Version consolidée au 20 juillet 2016. Recuperado de: https://www.legifrance.gouv.fr/affichTexte.do?cidTexte=JORFTEXT0000006934 $51 \&$ categorieLien=id

6 Sentencia de la Cour de Cassation de 1 de julio de 1970. 
Para los tribunales de los Estados Unidos, a la originalidad se llega gracias a la noción de variación distinguible (distinguishable variation ${ }^{7}$ ), según la cual una obra la ostenta cuando las aportaciones del autor suponen una variación apreciable, y no meramente trivial, con respecto a las obras preexistentes o a la realidad.

Un apunte interesante, extraído de los sistemas anglosajones (británico, australiano y neozelandés), es el concepto de protección global de la obra. Por ejemplo, en la obra audiovisual el objeto de protección es la película, entendida como grabación audiovisual. Las contribuciones realizadas para la misma pueden ser protegidas como obras autónomas, como puede ocurrir con el guion (obra literaria) o la banda sonora (obra musical). Sin embargo, se protege el conjunto, la grabación audiovisual en sentido amplio (González, 2001: 39), y su particular visión de la originalidad. Esto es extrapolable a la música, donde aparecen agentes con aportes creativos diferentes, pero todos necesarios para su creación.

Por último, las Directivas Comunitarias ${ }^{8}$ concernientes a propiedad intelectual (Pulido et al., 2016: 3), defienden una posición de la novedad, más que el criterio de originalidad subjetiva y, por tanto, no presta tanta atención a la personalidad que pueda aportar el autor.

Las visiones estadounidense y francesa son las más cercanas a la tesis que se defiende en el presente artículo, ya que insisten en los requisitos de originalidad y creatividad, básicos para poder proteger a todo potencial autor.

\section{a) Originalidad subjetiva y flamenco}

Es fundamental esbozar algunos elementos del flamenco y analizar el concepto de originalidad dentro del mismo para un mejor entendimiento del estudio. El flamenco es un género musical abierto que se distingue por su flexibilidad (Steingress 2002:45). Las temáticas, armonías, instrumentación, que en un principio seguían unos patrones muy marcados, cada día son más moldeables a nuevos recursos (Manuel 2010:114). Por ello, es muy dado a la fusión con otros géneros y es considerado como un género musical vivo (Bermúdez y Pérez 2009:131). Dada su importancia, en 2010 es aceptado en la Lista Representativa del Patrimonio Cultural Inmaterial de la Humanidad por la UNESCO 9 .

Su riqueza y variedad se observan desde una doble perspectiva: por un lado, desde las culturas de las que tomó elementos -andaluza, gitana, árabe, judía- (Romero, 1996: 75); por otro lado, desde sus tres tipos de manifestaciones: cante, baile y toque (Infante, 1980: 27; Palma et al., 2017: 59). Cuenta con un componente regional unido a Andalucía (Cruces, 2001: 16; Aoyama, 2009: 81), donde existen estilos por regiones, ciudades o incluso barrios (Manuel, 2010: 108). Además, se caracteriza

7 Circular 92, Copyright Law of the United States and Related Laws Contained in Title 17 of the United States Code. Dec. 2012. Recuperado de: https://www.copyright.gov/title17/ Circular 96. Code of Federal Regulations, Title 37- Patents, Trademarks, and Copyrights, Copyright Office regulations codified in the Code of Federal Regulations (CFR). Recuperado de: https://www. copyright.gov/title37/202/.

8 Directiva 2001/29/CE, de 22 de mayo - LCEur|2001/2153. DERECHO DE AUTOR. Armonización de determinados aspectos de los derechos de autor y derechos afines a los derechos de autor en la sociedad de la información y Directiva 2011/77/UE del Parlamento Europeo y del Consejo de 27 de septiembre de 2011 por la que se modifica la Directiva 2006/116/CE relativa al plazo de protección del derecho de autor y de determinados derechos afines. Diario Oficial de la Unión Europea, 10 de octubre de 2011.

9 La UNESCO con dicha lista representativa lo que persigue es la salvaguardia del patrimonio cultural no tangible. 
por la tradición oral que refleja su repertorio, a menudo nutrido por obras populares situadas en el dominio público ${ }^{10}$ (Calvo y Gamboa, 1994: 208). Otra de las características de su repertorio, es que se conocen los cantes o palos ${ }^{11}$ por las personas que los ejecutan y le dan su sello propio, desconociéndose en muchos casos su origen exacto. Ejemplos significativos son las seguiriyas de Antonio Mairena o las soleares de Tomás Pavón.

Los elementos estructurales del flamenco que pueden reflejar la originalidad subjetiva, desde una perspectiva de los derechos de autor, son los enunciados por Rosón (2010: 29): armonía, letra, melodía y ritmo. Estos elementos han sido refrendados por entrevistas a expertos ${ }^{12}$ del flamenco. Siendo, a priori, protegibles letra y melodía, ya que armonía y ritmo, son elementos que provienen en su mayoría del palo que se ejecute y se encuentran en el dominio público.

En este artículo, se plantea la interpretación como quinto elemento estructural ${ }^{13}$, pudiendo llegar a hacer equivalentes la creatividad del autor y el talento del intérprete (Towse, 2007: 749; Bryant y Throsby, 2006: 522).

\section{Un modelo para la estimación del valor de la obra flamenca}

El valor cultural "se expresa a través de procedimientos comunes y juicios colectivos, comunicados por los medios de comunicación a través de aplausos de la audiencia, comentarios de expertos, premios, o la longitud del texto y las imágenes en medios de comunicación impresos y electrónicos" (Hutter y Frey, 2010: 205).

A partir del modelo presentado en este apartado se ofrece un instrumento metodológico para la estimación del valor (Klamer, 2005: 465), que el intérprete flamenco aporta a la obra. La aproximación se realiza en términos culturales y económicos y permite observar el valor añadido por el intérprete a la obra, no reconocido por los derechos de autor. Para ello, se estima el valor cultural a partir de la ecuación [1], donde aparecen los agentes que intervienen en la creación de la obra -palo, autor e intérprete- y sus aportes creativos materializados en los elementos estructurales de la obra.

$$
\text { Valor Cultural }=\beta 1+\beta 2+\beta 3^{*}(1+x 1+x 2 \ldots+x n)=\beta 1+\beta 2+\beta 3^{*}\left(1+\sum x n\right)[1]
$$

- $\beta 1$ : Parámetro que refleja el valor cultural añadido por el palo. Dentro del mismo se encuentran dos elementos estructurales fijos en la obra: ritmo y armonía (Throsby, 1983: 68; Rosón, 2010: 32; Manuel, 2010: 111). Estos elementos se sitúan en el dominio público.

10 Dominio público se refiere al patrimonio cultural que está libre de exclusividad en su acceso y uso (Landes y Posner, 1989: 344; Geiger, 2017: 99)

11 El "palo" es cada una de las variedades tradicionales del cante flamenco, como son: la seguiriya, la soleá, las bulerías o el fandango, entre otras. Estas variedades se distinguen por tener una métrica propia, denominada "compás" y una armonía delimitada para el acompañamiento (Manuel, 2010: 111).

12 Siguiendo la definición dada por Holbrook (1999a) se entiende por experto a aquella persona que, a través de un entrenamiento especializado y de su experiencia acumulada, cuenta con un alto grado de conocimiento artístico. En el caso del flamenco un ejemplo claro podría ser las personas dedicadas a la crítica o a la investigación.

13 Esta idea está sustentada en la bibliografía existente: Romero (1996: 75), Towse (2007:749) y en las entrevistas realizadas a los expertos, donde se destacó el aporte creativo del intérprete, concretado en los elementos que reflejan la originalidad subjetiva. 
- $\beta 2$ : Parámetro que refleja el valor cultural aportado por el autor, mediante dos elementos estructurales, también fijos: melodía y letra (Werck y Heyndels, 2007: 28; Rosón, 2010: 30). Dichas aportaciones se protegen mediante derechos de autor $^{14}$ (Manuel, 2006: 128).

- $\beta 3$ : Parámetro que refleja el valor cultural agregado por la ejecución del intérprete, ciñéndose exactamente a lo que dicta el autor con sus indicaciones o la partitura en caso de existir. Es muy importante este parámetro, porque se debe reconocer el valor cultural aportado por los intérpretes aun no añadiendo originalidad o altura creativa extra a la obra con su trabajo. Para ello, se garantiza su existencia multiplicándolo siempre por una cantidad $\geq 1$. Se protegen mediante Derechos vecinos ${ }^{15}$.

- $(\mathrm{x} 1+\ldots+\mathrm{xn})$ : Representan los elementos identificables que materializan las aportaciones creativas realizadas por los intérpretes (Towse, 2007: 748). No están protegidos por ninguna figura actual de derecho de propiedad intelectual.

Al analizar la protección otorgada a autor e intérprete, se observa un tratamiento marginal para el trabajo creativo realizado por el segundo. De lo que se desprende menor remuneración económica y menor protección de dicho trabajo creativo. En concreto, ¿cómo identificar el valor cultural agregado por el intérprete a la obra flamenca? En el siguiente apartado se identifican los elementos clave.

a) El valor añadido por el intérprete: $\beta 3 *\left(1+\sum x n\right)$

Observando la Ecuación [1], $\left(1+\sum x n\right)$ incluiría todos los elementos distintivos aportados por el intérprete que agregan valor cultural de la obra ${ }^{16}$ (Hadida, 2010: 50; Levinson, 2015: 76). A continuación se expone una lista no cerrada de estos elementos:

- x1: Improvisación ${ }^{17}$

- x2: Carácter propio del artista

- x3: Duende ${ }^{18}$

- $x 4$ : Técnica $^{19}$

14 Los derechos de autor incorporan los morales de paternidad, integridad, divulgación, modificación, retirada del comercio por medio de convicciones intelectuales o morales y acceso a ejemplar único o raro (art. 14). En cuanto a los patrimoniales, la Ley otorga los derechos de reproducción (art. 18), distribución (art. 19), comunicación pública (art. 20) y trasformación (art. 21).

15 Los Derechos vecinos hacen referencia a los morales de paternidad e integridad de los artistas (art. 113 TRLPI) y a los patrimoniales de reproducción (art. 18), distribución (art. 19) y comunicación pública (art. 20).

16 Además de la bibliografía utilizada, las variables enumeradas a continuación fueron extraídas de las preguntas 1 y 2 de las entrevistas realizadas a los expertos. En el Anexo II aparecen las preguntas utilizadas para efectos del presente artículo.

17 Según el Diccionario de la Real Academia Española (RAE), improvisar es "Hacer algo de pronto, sin estudio ni preparación". Obviamente, la improvisación solo la puede ejecutar el intérprete al salirse de lo estipulado por el autor en sus indicaciones o en la partitura en caso de existir.

18 Según Salazar (2015: 64) el duende "es transmitido, como una emoción, por el que ejecuta un paso, un toque o un cante al que lo ve y, además, es una experiencia subjetiva que marca a quien lo posee, y que le identifica ante los demás".

19 Gama de recursos que utilizan los artistas, independientemente de la disciplina a la que pertenezcan (baile, cante o toque), para llevar a cabo su interpretación. Por ejemplo, en el cante algunas de las más utilizadas son: la aspiración de la faja abdominal, activación de la cara, muecas, entre otros (Márquez, 2017: 5). 
- x5: Lugar en el que se realiza la interpretación ${ }^{20}$.

- x6: Valor de marca ${ }^{21}$

- x7: Reconocimiento por parte del público 22 .

- x8: Reconocimiento por parte de expertos.

- xn: Cualquier otro elemento que agregue valor cultural de la obra.

Si se relacionan los elementos distintivos enumerados con el concepto de originalidad subjetiva presentado anteriormente, se observan las siguientes relaciones:

- Altura creativa ${ }^{23}(x 2, x 4)$

- Variación distinguible ${ }^{24}(\mathrm{x} 1)$

- Novedad ${ }^{25}$ (x1)

- Espíritu Humano ${ }^{26}$ (x3)

La conclusión que se extrae es que $\sum x n$ se corresponde con la idea de originalidad subjetiva o altura creativa (Varian, 2005: 124). En el caso de que algún $x 1, \ldots . x n$, sea distinto de cero, el intérprete aporta un valor adicional a la obra, además del valor añadido por el autor. En la normativa vigente en España, y en otros muchos países, no es reconocido. El problema real surge cuando: $\beta 1+\beta 2 \leq \beta 3^{*}\left(1+\sum x n\right)$. Este caso sucede en géneros musicales donde el intérprete tiene un gran peso específico, como pasa en el flamenco, la salsa o el jazz.

La cuestión es dilucidar cómo el trabajo creativo realizado por el intérprete es protegido y recibe una contraprestación por el valor que añade a la obra. Hasta el momento, la protección es parcial mediante los derechos vecinos y la contraprestación depende del reconocimiento que le permite realizar conciertos y tener un caché en consecuencia. Sin embargo, los derechos de autor (Plant 1934: 26; Landes, 2005: 100), en la normativa española y de otros países no ayudan al reconocimiento jurídico de la aportación creativa que realiza el intérprete, que es fundamental para la obra flamenca.

\section{b) Características que capturan el valor agregado por el intérprete}

Para definir el valor agregado por el intérprete se parte de la multidimensionalidad del mismo (Gallarza y Gil, 2006: 35; Icazurriaga et al., 2016: 10). Esto afecta a la creación de la marca del intérprete (Aaker y Keller, 1990: 7; Hernando, 2017b: 46) que es un elemento definitorio del bien creativo, influyendo en la elección de los consumidores (Kapferer y Shuiling, 2004: 98).

El intérprete puede influir en varias manifestaciones del valor cultural como son:

- Valor estético: Siendo la música un arte, posee características propias que la diferencia de otros bienes: las artísticas (Holbrook, 1999b: 148). Este tipo de cualidades se observan en el intérprete que añade originalidad subjetiva a la obra (Holbrook y Hirchman, 1982: 136; Hernando, 2017a: 36).

20 Festivales y teatros de reconocido prestigio.

21 La marca del artista acentúa la diferenciación e influye en la decisión de compra o de asistencia a espectáculos de los aficionados (Hernando, 2015: 57).

22 Con público se entiende a los consumidores de grabaciones y espectáculos en vivo de flamenco.

23 La doctrina alemana incorpora el concepto Gestaltungshöhe como requisito para llegar al concepto de originalidad subjetiva.

24 Según los tribunales norteamericanos, a la originalidad se llega a partir de esta noción de variación distinguible.

25 Concepto aplicable a la normativa francesa y a la norteamericana.

26 Apunte destacado por Loewenheim (de Román, 2003) y por Romero (1996). 
- Valor cognitivo: Dentro de la creación artística es muy importante la comprensión del concepto del músico y su obra (Holbrook y Hirchman, 1982: 137; Au et al., 2016: 30). Al ser el intérprete la persona que exterioriza la obra (Felton, 1978: 43), es el encargado en última instancia de que el mensaje que guarda la obra se entienda y se transmita a la audiencia. También se puede observar en la técnica o estilo que use para manifestar el mensaje que dan lugar al estudio y evolución del flamenco (Márquez, 2017).

- Valor emocional: Será su imagen uno de los factores determinantes para hacer ver a los consumidores el valor de la música como un sentimiento donde reflejan su identidad (Bermúdez y Pérez, 2009: 28; Hernando, 2015: 43). Siguiendo la línea marcada por Throsby (2003: 279), "el arte es una manifestación de la naturaleza humana ${ }^{27 "}$ que se materializa gracias a los artistas.

- Valor de entretenimiento: Según Holbrook y Hirchman (1982), el valor de la diversión es fundamental para la experiencia de consumo. Se considera un placer hedónico derivado de actividades lúdicas, como son los conciertos o la escucha de grabaciones (Lazzaro, 2006: 15; Icazurriaga et al., 2016: 10).

- Valor de posesión: los aficionados y consumidores de música mantienen un deseo exclusivo de poseer una obra, como obra de arte que es (Frey, 2000: 17; Hernando, 2015: 44), siendo en muchos casos el intérprete la figura determinante.

- Valor simbólico/social: en el caso del flamenco, es algo palpable, ya que muchos intérpretes flamencos en la época de la posguerra española dejaban su lugar de origen para dedicarse profesionalmente al ámbito de la música en tablaos. De esta forma conseguían un sustento económico además de reconocimiento proyectado hacia el público (Hernando, 2015: 49). El papel del flamenco en general y del intérprete en particular, contaba entonces con una capacidad socio-cultural integradora (Cruces, 2000: 148).

- Valor de legado: Este concepto se adapta desde el punto de vista de la necesidad de legar a futuras generaciones sus contribuciones al estilo (Frey, 2000: 16; Hernando, 2015: 53).

Las dimensiones explicadas en este apartado son jerarquizables, siendo diferentes para cada caso. Para unos puede tener mayor importancia la dimensión emocional que la dimensión estética, por ejemplo: un intérprete con pocas facultades físicas para realizar determinados cantes y, sin embargo, que sea poseedor de una gran carga emocional que llegue al público. De esta forma, se deja la puerta abierta a futuras investigaciones sobre dicha jerarquización de las dimensiones del valor cultural en la obra musical flamenca (Heredia-Carroza et al., 2017b: 17).

Otro factor importante es el hecho de que el valor percibido del intérprete no es estático, ya que existen cambios en los gustos del mercado. Lo que hoy tiene un escaso valor cultural y económico, en el futuro puede tenerlo. Ejemplos claros son los de Camarón de la Isla y Enrique Morente con los álbumes La Leyenda del Tiempo u Omega, respectivamente. No tuvieron buena aceptación por parte de los aficionados cuando salieron al mercado. Sin embargo, hoy día son considerados álbumes

27 Dicha naturaleza humana es social y se desprende de las "creencias, tradiciones, costumbres, entre otras" (Throsby, 2003: 279). En el valor emocional captado por el consumidor, además del fundamental papel del intérprete, aparecen otros factores como son el entorno, la localidad donde se realice, los parientes o amigos con los que asista al evento, pero para efectos de este artículo solo se explica la contribución del intérprete. 
conceptuales que transgredieron con los paradigmas existentes hasta ese momento ${ }^{28} \mathrm{y}$, ambos cantaores tienen un gran reconocimiento por parte del público y de los expertos. Por ello, se hace necesaria la perspectiva temporal como variable imprescindible para poder visualizar las dimensiones enumeradas. De esta forma se podrá observar "la supervivencia en el tiempo" de las obras y de los artistas (Simonton, 2014: 20; Heredia-Carroza et al., 2017c: 14).

Todo ello influye en la consolidación de la marca propia del intérprete, elemento que acentúa la diferenciación de los bienes culturales (Caves, 2003: 80). La misma otorga un valor utilitario y simbólico a dichos bienes e influye en la decisión de compra del consumidor (Yoo y Donthu, 2001: 2). La marca da información acerca del producto, aportando utilidad al consumidor y reduciendo la incertidumbre acerca de sus características (Kapferer y Schuling, 2004: 100), aumentando de esta forma la calidad del bien (Throsby, 1983: 65; Hagtvedt et al., 2008: 206; Zieba y O'Hagan, 2010: 156).

La Figura 1 ayuda a una mejor interpretación metodológica del modelo planteado.

Figura 1

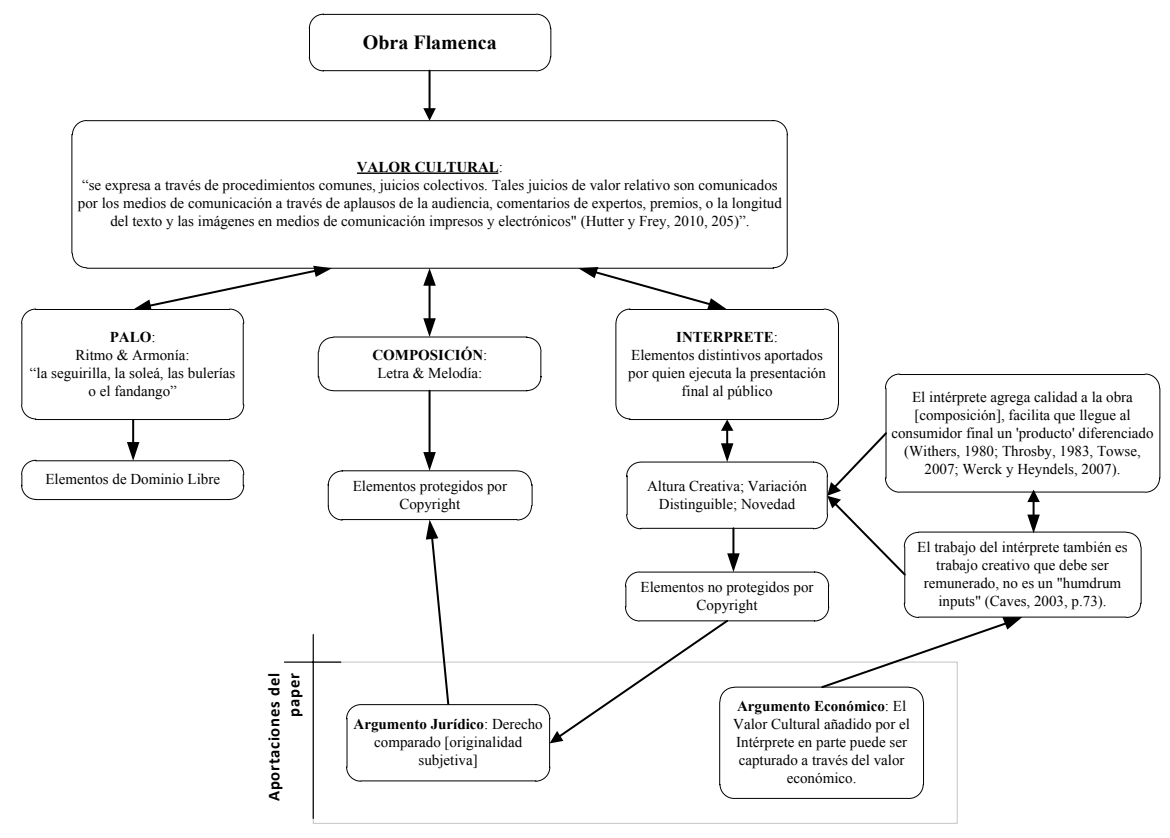

Fuente: Elaboración Propia

\section{Análisis de casos}

Tras la explicación del modelo, se analizan dos casos que ayudan a sustentar la argumentación sostenida durante todo el artículo. La modelización se muestra, de nuevo, a partir de la ecuación [1] ${ }^{29}$.

28 Se comienza a grabar canciones no cantes como se hacía anteriormente.

29 Siendo la regla general el modelo propuesto en la ecuación [1], en este apartado se observará cómo es posible moldearla para casos particulares. 


\section{Valor Cultural $=\beta 1+\beta 2+\beta 3^{*}\left(1+\sum x n\right)[1]$}

Como se ha visto, todo valor cultural, no tiene por qué tener asociado un valor económico, como pasa con $\beta 1^{30}$, no obstante, ontológicamente, todo valor económico, fuente de derechos, tiene que estar asociado a un valor cultural.

El primer caso se presenta para una posible solución a la problemática que dan otros ordenamientos jurídicos y tribunales, en este caso el francés. El segundo caso es el de Camarón de la Isla, donde se justifica gracias al aporte extra de calidad la opción de considerar al intérprete como autor en colaboración de la obra.

\section{a) Manitas de Plata}

Un ejemplo claro, y de interés para el estudio realizado, es el recogido en la Sentencia emitida por la Cour de Cassation francesa, el 1 de Julio de 1970, con el caso Manitas de Plata.

En 1960, en un peregrinaje de gitanos de Saintes-Maries-de-la-Mer, Manitas de Plata interpretó a la guitarra un determinado número de obras que fueron registradas por la sociedad Paris-Records, editando los discos "Président". En julio de 1964, el disco realizado a partir de este registro fue producido por la sociedad Stereo-Press y puesto a la venta por Paris- Record, sin la autorización del guitarrista.

El caso se llevó a los tribunales franceses donde una experta, mediante instancia, aseguraba que las obras musicales interpretadas presentaban el carácter de una creación personal, lo que permitía considerar a Manitas de Plata como autor de su interpretación.

De la sentencia final se extraen conclusiones, tales como el tratamiento especial que tiene el flamenco. Se puede observar la idea de originalidad subjetiva cuando en la sentencia se refiere a "la inspiración y el aire popular del flamenco" (Steingress, 2007: 48). El avance más importante es el hecho de considerar al guitarrista autor al añadir su temperamento y estilo propio, lo que la Cour de Cassation consideró "presenta un carácter original".

Este caso es especial debido a que las obras interpretadas por Manitas de Plata son totalmente improvisadas en su origen, con lo que en el modelo, $\beta 2=0$, no existiendo autor, con lo que el término que captura la aportación del intérprete quedaría, $\beta 3^{*}\left(0+\sum x n\right)$. Por lo tanto, el valor cultural final vendría expresado por la ecuación [2]:

\section{Valor Cultural final: $\beta 1+\beta 3^{*} \sum \times n[2]$}

- Existe el valor cultural aportado por el palo o forma musical utilizada ( $\beta 1)$.

- No hay aportación del autor, puesto que en una improvisación no hay partitura ni indicaciones preexistentes sobre la que interpretar. $(\beta 2=0)$

- Tampoco existiría el escalar 1 dentro de la multiplicación con $\beta 3$, al existir libertad absoluta en la improvisación.

- Quedando $\sum x n$, como valor cultural agregado por el intérprete, donde según la sentencia referida las variables serían:

- x1: improvisación

- x2: inspiración

- x3: aire popular y folclórico del flamenco, aportado por el artista

- x4: temperamento

30 Formas e ideas musicales, como son el palo flamenco (Encabo y Rosón, 2011: 55). 
- $x 5$ : estilo propio

- $\mathrm{xn}$ : otros

Se observa que el valor cultural de la obra, de Manitas de Plata en este caso, al ser improvisada, reside en el palo interpretado, y en la aportación del guitarrista, que sin tener una melodía preestablecida sobre la que interpretar, crea una obra con entidad propia. Esto lo convierte, en términos de valor económico, en el único posible perceptor del mismo, como titular de los derechos de autor, y de todo lo que se desprende de ellos.

\section{b. Camarón de la Isla}

Camarón de la Isla es considerado uno de los mejores cantaores flamencos de la historia reciente, además de un gran transgresor, abriendo nuevas vías de interpretación (Gamboa y Núñez, 2003: 93). Pocas semanas antes de su muerte, en su última entrevista televisada, hacía referencia a su situación acerca de los derechos de autor, que no poseía, sobre las obras en las que había colaborado (Sáenz, 1992: 1).

Se plantea un problema de repercusiones legales -se cuestiona la validez de firmas reconocidas como autoras de canciones-, morales (de Román, 2008: 233) -se reivindica la creación del intérprete junto al compositor-y culturales -se descubre el escaso interés oficial, industrial, que no popular, por la obra de un artista reconocido como único (Schulze, 2005: 401; Connolly y Krueger, 2006:34; Towse, 2010:306).

El detonante de estos hechos fueron los derechos que puede generar la obra de Camarón, una obra sobre la que el cantaor participa como autor en un bajo porcentaje. Oficialmente, publicó 17 discos, además de dos recopilaciones. Son 164 canciones, de las que José Monje Cruz, tal es el nombre del artista, aparece como autor en los registros de la Sociedad General de Autores de España (SGAE) sólo en 27 (Saenz de Tejada, 1992: 1; Manuel, 2010: 125).

El incuestionable reconocimiento del valor cultural aportado por el cantaor a las obras desde el punto de vista de la calidad, no se traduce en un reconocimiento de valor económico materializado en derechos, considerándose de esta manera un humdrum imput (Caves, 2003: 73).

Existe un valor cultural aportado por el palo, compositor y, por supuesto, por el intérprete. Lo que hace especial a este caso es la figura del intérprete, que hace que la obra sea única, y su valor cultural aumente de forma notable, ya que Camarón es considerado una superestrella (Pitt, 2010: 227). Camarón de la Isla es sin duda uno de los mejores cantaores de la historia, gracias a dos principios: rareza y belleza de su talento (Cox et al., 1995: 335; Towse, 2007: 747); de hecho se han interpretado las mismas obras por parte de otros artistas, y nunca han llegado a generar el mismo nivel de ventas (Hansmann y Santilli, 2001: 276; Meiseberg, 2014: 13). Esta realidad quedaría reflejada en la Ecuación [3].

$$
\beta 1+\beta 2+\beta c^{31 *}\left(1+\sum x n\right) \geq \beta 1+\beta 2+\beta 3^{*}\left(1+\sum x n\right)[3]
$$

Otro de los escenarios que se pueden plantear, es qué sucede cuando el valor cultural añadido por el intérprete es mayor que el aportado por el autor, tal y como aparece en la ecuación [4]:

31 Parámetro relativo a la interpretación de Camarón de la Isla. 


\section{$\beta 1+\beta 2 \leq \beta c^{*}\left(1+\sum x n\right)[4]$}

La interpretación es la que hace decantarse al consumidor por la compra de una entrada a un espectáculo o de música grabada (Heredia-Carroza et al., 2017a: 10), actuando la interpretación como un elemento diferencial de calidad (Caves, 2002: $73)$. En casos como este, la gran aportación de originalidad subjetiva ( $\left.\sum x n\right)$ por parte del intérprete, podría servir para fundamentar la posibilidad de considerar al intérprete creativo como coautor de la obra.

\section{Conclusiones}

Es la relación de afinidad, conexión o vecindad entre los derechos de los artistas y los derechos de autor lo que provoca la problemática analizada en este artículo en legislaciones como la española. Desde un punto de vista extrajurídico, esa relación expresa como los objetos sobre los que recaen los derechos de los titulares del Libro II del TRLPI, necesitan para poder existir de la preexistencia de los autores y sus trabajos. El art. 105 del mismo cuerpo legal trata el lazo de unión entre la ejecución artística y la obra sobre la que se proyecta.

Según Bercovitz (2007: 1643), la afinidad entre intérpretes y autores es tal, que debería preverse para aquéllos una protección equivalente a la de éstos. Como se ha defendido a lo largo de todo el artículo, los artistas desarrollan una labor intelectual que involucra aspectos como son impronta, personalidad, imagen, marca, entre otras, a través de sus aportaciones. Todo ello de una naturaleza creativa diferente a las tareas desarrolladas por un productor o una entidad de radiodifusión, de carácter eminentemente empresarial.

Se debe reseñar, que una ejecución artística crea un bien inmaterial, que nace en el momento que una persona exterioriza la obra. El bien inmaterial creado será distinto a cualquier interpretación realizada sobre la misma obra por otra persona, por similares que puedan resultar (Towse, 2007: 751). Con lo que se llega a la conclusión de que surgirán tantos bienes inmateriales como artistas haya (Bercovitz, 2007: 1643).

De esta forma, puede haber imitaciones a una determinada interpretación, siendo un producto copiable, pero no reproducible debido a su originalidad. Este es el argumento para considerarla protegible mediante derechos de autor que es el mecanismo usado para ello en la actualidad. Pudiendo aparecer en el futuro otros modelos de negocio para capturar el valor económico o para remunerar el trabajo creativo del intérprete.

Siguiendo la tesis de Caves (2003), la interpretación no podría ser considerada como un mero humdrum imput, no siendo un acto rutinario dentro de la cadena de producción artística (Heredia-Carroza et al., 2017a: 4). Por lo tanto, tendría que ser remunerada con unos derechos de autor acordes a su importancia. De esta forma el artista podría capturar a través del valor económico el valor cultural que aporta a la obra.

Finalmente, gracias a un estudio de Derecho Comparado, se propone que, en los casos en los que el valor cultural aportado por el intérprete sea determinante, a éste se le considere autor en colaboración.

Según el art. 7.1 TRLPI, una obra en colaboración consiste en un trabajo en el que todos los autores aportan una parte y se consigue un resultado unitario. Es evidente que en casos como el de Camarón de la Isla, la interpretación no puede disociarse de la obra del compositor, forman un todo que incluye sus elementos estructurales. 
En definitiva, la obra viene caracterizada por la interpretación; otra interpretación de la misma obra la desvirtuaría como tal, sería una obra distinta.

Todo ello está en consonancia con los joint works ${ }^{32}$ de la legislación norteamericana, al considerar que intérpretes y compositores realizan un trabajo con un resultado unitario, aportando conjuntamente un alto valor a la obra. Esto derivaría en un aumento de los derechos de autor, propiciado por el valor económico que la obra genera.

\section{Bibliografía}

Aaker, D. A. \& Keller, L. (1990). "Consumer evaluations of brand extensions". The Journal of Marketing, 54 (1), 27-41. Doi: 10.2307/1252171.

Aoyama, Y. (2009). "Artist, Tourists, and the State: Cultural Tourism and the Flamenco Industry in Andalusia, Spain". International Journal of Urban and Regional Research, 33 (1), 80-104. Doi: 10.1111/j.1468-2427.2009.00846.x.

Bercovitz, R. (2007). Comentarios a la Ley de Propiedad Intelectual, $3^{\text {a }}$ ed. Madrid. Tecnos.

Bermúdez S. \& Pérez, J. (2009). "Introduction: Spanish Popular Music Studies”, Journal of Spanish Cultural Studies, 10(2), 127-133. Doi: 10.1080/14636200902990661.

Bryant, W. D. A. \& Throsby, D. (2006). "Creativity and the behavior of artists". En Ginsburg, V. A. and Throsby,D. (Eds.), Economics of Art and Culture (p. 507-530). North Holland.

Calvo, P. \& Gamboa, J. M. (1994). Historia-Guía del Nuevo Flamenco: El Duende de Ahora. Madrid: Ediciones Guía de Música.

Caves, Richard E. (2003) "Contracts between Art and commerce". The Journal of Economic Perspectives, 17(2), 73-84. Doi: 10.1257/089533003765888430.

Circular 92, Copyright Law of the United States and Related Laws Contained in Title 17 of the United States Code. Dec. 2012. Recuperado de: https://www.copyright.gov/title17/

Circular 96. Code of Federal Regulations, Title 37- Patents, Trademarks, and Copyrights, Copyright Office regulations codified in the Code of Federal Regulations (CFR). Recuperado de: https://www.copyright.gov/title37/202/.

Connolly, M. \& Krueger A. B. (2006). "Rockonomics: The economics of Popular Music". En Ginsburgh, V. A. and Throsby (Eds.). Economics of Art and Culture. Elsevier. Doi: $10.3386 / \mathrm{w} 11282$.

Cruces Roldán, C. (2000). "El Flamenco", en Gran Enciclopedia de Andalucía, Siglo XXI, Sevilla: Ediciones Tartessos.

Cruces Roldán, C. (2001). El flamenco como Patrimonio. Anotaciones a la Declaración de la voz de la Niña de los Peines como bien de interés cultural, Sevilla: Bienal de Arte Flamenco.

de Román, R. (2003). Obras musicales, compositores, intérpretes y nuevas tecnologías. Madrid. REUS.

de Román, R. (2008). "Una visión particular de los derechos morales de los artistas intérpretes o ejecutantes", Revista General de Legislación y Jurisprudencia, 2(marzo),

32 Code of Federal Regulations, Title 37- Patents, Trademarks, and Copyrights, Copyright Office regulations codified in the Code of Federal Regulations (CFR). These regulations are also known as Circular 96. 
233-258. Recuperado de: http://www.editorialreus.es/revistas/revista-general-de-legislacion-y-jurisprudencia/una-vision-particular-de-los-derechos-morales-de-los-artistas-interpretes-o-ejecutantes/12/

de Román, R. (2010). "Derecho de propiedad intelectual de los artistas intérpretes o ejecutantes como derecho análogo al derecho de autor". Diario La Ley, no. 7987. LA LEY. Recuperado de: https://dialnet.unirioja.es/servlet/articulo?codigo=3186011.

Directive 2001/29/EC, of the European Parliament and of the Council of 22 May 2001, on the harmonization of certain aspects of copyright and related rights in the information society. Recuperado de: http://eur-lex.europa.eu/legal-content/ES/TXT/PDF/?uri=CEL EX:32001L0029\&from $=E N$

Directive 2006/116/EC of the European Parliament and of the Council of 12 December 2006 on the term of protection of copyright and certain related rights. Recuperado de: http://eur-lex.europa.eu/legal-content/ES/TXT/HTML/?uri=CELEX:32006L0116\&from $=\mathrm{EN}$.

Encabo, M. A. \& Rosón, T. (2011). "Ideas y su concreción en el ámbito musical”. En Ortega, J. (Ed.), Ideas, Bocetos, Proyectos y Derechos de autor, (p. 55-76). Madrid. REUS.

Felton, .M. V. (1978) "The economics of the creative arts: the case of the composer". Journal of Cultural Economics, 2(1), 41-61.

Frey, B. (2000). La economía del arte. Barcelona. Colección estudios económicos.

Gamboa, J. M. and Núñez, F. (2003). Camarón vida y obra. Iberautor Promociones Culturales.

Gallarza, M. G. \& Gil, I. (2006). "Desarrollo de una escala multidimensional para medir el valor percibido de una experiencia de servicio". Revista española de investigación de marketing, 10(2), 25-59. Recuperado de: http://www.esic.edu/documentos/revistas/ reim/070704_185800_E.pdf.

Geiger, C. (2017). "Copyright as an access right: Securing cultural participation through the protection of creators'interests". En Rebecca Giblin and Kimberlee Weatherall (Eds.), What if we could reimagine copyright, (p. 73-109). ANU Press.

González, Alfonso. (2001) "La noción de obra audiovisual en el derecho de autor". Revista de propiedad intelectual, 7(enero - abril), 9-70. http://moodle2.unid.edu.mx/dts_cursos_mdl/lic/CYTC/ALC/AM/10/Nocion.pdf

Hadida, A. L. (2010). "Commercial success and artistic recognition of motion picture projects" Journal of Cultural Economics, 34(1), 45-80. Doi: 10.1007/s10824-009-9109-z.

Hagtvedt, H., Hagtvedt, R. \& Patrick, V. M., (2008). "The perception and evaluation of visual art”. Empirical Studies of the Arts, 26 (2), 197-218.

Hansmann H. \& Santilli M. (2001). "Royalties for Artists versus Royalties for Authors and Composers". Journal of Cultural Economics 25(4), 259-281. Doi:10.1023/A:1017923625973.

Heredia-Carroza, J., Palma Martos \& Aguado, L. F. (Agosto, 2017). "Valor aportado por el intérprete a la obra musical y retribución de derechos de autor. El caso del flamenco en España". I Seminario Iberoamericano de Economía de la Cultura. Universidad Nacional de Colombia, sede Medellín, Medellín, Colombia. 
Heredia-Carroza, J., Palma Martos \& Aguado, L. F. (Marzo, 2018). "Distinguishable elements of the flamencgo musical work. An empirical approach". VIII Workshop en Economía y Gestión de la Cultura (8WCEM). Universidad de Sevilla, Sevilla, España.

Heredia-Carroza, J., Palma Martos, L. \& Aguado, L. F. (2017). "Flamenco y Derechos de Autor. El caso de Camarón de la Isla". Trabajo en curso.

Hernando, E. (2015). El valor percibido del arte: desarrollo de una escala de medición. (Tesis doctoral). Universidad Autónoma de Madrid, Madrid. Recuperado de: https://repositorio.uam.es/bitstream/handle/10486/663322/hernando_calero_elisa. pdf? sequence $=1$

Hernando, E. \& Campo, S. (2017). "Does the Artist's Name Influence the Perceived Value of an Art Work?" International Journal of Arts Management, 19(2), 49-58.

Hernando, E. \& Campo, S. "An artist's perceived value: development of a measurement scale". International Journal of Arts Management, 19(2), 33-47.

Holbrook, M. B. \& Hirschman, E. (1982). "The experiential aspects of consumption: consumption fantasies, feelings and fun”. Journal of consumer research, 9(2), 132-140.

Holbrook, M. B. (1999) "Popular Appeal versus Expert Judgments of Motion Pictures". Journal of consumer research, 26(2), 144-155. Doi: 10.1086/209556.

Holbrook, M. B. (1999) Consumer value: a framework for analysis and research: Interpretative market research series. London and New York. Routledge.

Hutter, M. \& Frey, B. (2010). "On the Influence of Cultural Value on Economic Value". Revue d'économie politique, 120(Jan), 35-46. Doi: 10.3917/redp.201.0035.

Icazuriaga, J., Cuadrado, M. \& Miquel, M. J. (Septiembre, 2016) "Analysing Perceived Value, Satisfaction and Pruchase Intention in the Music Industry". 7th Vienna Music Business Research Days, Vienna. University of Music and Performing Arts Vienna, Viena, Austria.

Infante, B. (1980). Orígenes de lo flamenco y secreto del cante jondo. Sevilla. Junta de Andalucía, Consejería de Cultura. Recuperado de: http://www.juntadeandalucia.es/ export/drupaljda/1337160241origenes_de_lo_flamenco.pdf

Kapferer, J. N. \& Schuilling, I. (2004). "Executive Insights: Real Differences between Local and International Brands: Strategic Implications for International Marketers". Journal of international marketing, 12(4), 97-112. Doi: 10.1509/jimk.12.4.97.53217.

Klamer, A. (2005). "Value of Culture". En Towse, R. (Ed). A Handbook of Cultural Economics" (p. 465-472). Edward Elgar Publishing.

Landes, W. M. \& Posner, R. A. (1989). "An Economic Analysis of Copyright Law”. Journal of Legal Studies 325(33), 344-353.

Landes, W. M. (2005). "Copyright”. En Towse, R. (Ed.). A Handbook of Cultural Economics (p. 100-113). Edward Elgar Publishing.

Lazzaro, E. (2006). “Assessing Quality in Cultural Goods: The Hedonic Value of Originality in Rembrandt's Prints". Journal of Cultural Economics, 30(1), 15-40. Doi: 10.1007/ s10824-005-1918-0.

Levinson, J. (2015). Musical Concerns, Essays in Philosophy of Music. Oxford. Oxford University Press. 
Loi relative aux droits d'auteur et aux droits des artistes-interprètes, des producteurs de phonogrammes et de vidéogrammes et des entreprises de communication audiovisuelle. Collection de lois accessible en ligne, $n^{\circ} 85-660$, du 3 juillet 1985 . Recuperado de: https://www.legifrance.gouv.fr/affichTexte.do?cidTexte=JORFTEXT000000693451 \&categorieLien=id

Manuel, P. (2006). "The Saga of a Song: Authorship and Ownership in the Case of "Guantanamera", Latin American Music Review 27(2), 121-147.Doi: 10.1353/lat.2007.0007.

Manuel, P. (2010). "Composition, Authorship and Ownership in Flamenco, Past and Present”, Ethnomusicology 54(1), 106-135.

Márquez Limón, R. (2017). La técnica vocal en el flamenco: fisionomía y tipología. (Tesis doctoral). Universidad de Sevilla, Sevilla. Recuperado de: https://idus.us.es/xmlui/ handle/11441/63972

Meiseberg, B. (2014). "Trust the artist versus trust the tale: performance implications of talent and self-marketing in folk music". Journal of Cultural Economics 38(1), 9-42. Doi: 10.1007/s10824-012-9196-0.

Pitt, I. L. (2010) "Superstars effects on royalty income in a performing rights organization". Journal of Cultural Economics, 34(3), 219-236. Doi:10.1007/s10824-010-9123-1.

Palma, L., Palma, M. L., Rodríguez, A., Martín, J. L. \& Cascajo, I. (2017). "Live Flamenco in Spain: A Dynamic Analysis of Supply, with managerial implications". International Journal of Arts Management 19(3), 58-70.

Plant A. (1934) "The economic aspect of copyright in books". Economica 1(2), 167-195.

Pulido, N., Palma, L, Borrero, D. (Junio, 2016). "Copyright regimes in the European Union. An analysis of the Directive about the harmonisation (2001) effectiveness". 19th International Conference on Cultural Economics. Universidad de Valladolid. Valladolid.

Real Decreto Legislativo 1/1996, de 12 de abril, por el que se aprueba el texto refundido de la Ley de Propiedad Intelectual, regularizando, aclarando y armonizando las disposiciones legales vigentes sobre la materia. Recuperado de: http://noticias.juridicas. com/base_datos/Admin/rdleg1-1996.html.

Romero Jiménez, J. (1996). La otra historia del Flamenco. Sevilla. Junta de Andalucía.

Rosón, T. (2010). "El Flamenco como obra musical, coreográfica y escénica. Las obras originales y derivadas: versiones, arreglos y utilización de las obras". En Castilla, M. (Ed.). El Flamenco y los Derechos de Autor (p.23-38). Madrid, REUS.

Saenz de Tejada, I. (2 de Agosto de 1992). La herencia de un príncipe. EL PAÍS, Recuperado de: http://elpais.com/diario/1992/08/02/cultura/712706402_850215.html.

Salazar López, E. (2015) "La huella psicológica del duende flamenco". La nueva Alboreá, 32(abril-junio), 64-66. Recuperado de: https://www.juntadeandalucia.es/cultura/ flamenco/content/la-huella-psicol\%C3\%B3gica-del-duende-flamenco-elvira-salazar1\%C3\%B3pez

Schulze, G. G. (2005) "Superstars". En Towse, R. (Ed.). A Handbook of Cultural Economics (p. 401-407). Edward Elgar Publishing.

Sentencia de la Cour de Cassation, 1ère chambre civile - "Manitas de Plata". 1 July 1970. 
Simonton, D. K. (2014) "Creative Genius in Literature, Music and the Visual Arts". En Ginsburgh, V. A. and Throsby, D. (Eds.). Handbook of the Economics of Art and Culture (p. 14-49). Elsevier.

Steingress, G. (2002), "El flamenco como patrimonio cultural o una construcción artificial más de la identidad andaluza", Revista Andaluza de Ciencias Sociales, 1, 43-64. Universidad de Sevilla.

Steingress, G., Flamenco postmoderno: entre tradición y heterodoxia: un diagnóstico sociomusicológico (escritos 1989-2006). Signatura, 2007, Sevilla.

Throsby, D. (1983). "Perception of quality in demand for the theatre". MAQUARIE University, School of Economic and Financial Studies 250, 65-82.

Throsby, D. (2003). "Determining the value of cultural goods: How much (or how little) does contingent valuation tell us?". Journal of Cultural Economics 27, 275-285.

Throsby, D. (2006). "An Artistic Production Function: Theory and Application to Australian Visual Artists". Journal of Cultural Economics 30(1), 1-14. .Doi: 10.1007/ s10824-005-9001-4.

Towse, R. (2005). A Handbook of Cultural Economics", Glasgow: Edward Elgar Publishing.

Towse, R. (2007). "The Singer or the Song? Developments in Performers' Rights from the Perspective of a Cultural Economist". Review of Law \& Economics, 3(3), 745-766. Doi:10.2202/1555-5879.1158.

Varian, H. R. (2005). "Copying and Copyright" School of Information Management and Systems. Department of Economics. University of California, Berkeley. Recuperado de: http://people.ischool.berkeley.edu/ hal/Papers/2004/copying-and-copyright.pdf

Werck, K. \& Heyndels, B. (2007). "Programmatic choices and the demand for theatre: The case of Flemish theatres". Journal of Cultural Economics, 31(25), 25-41. Doi: 10.1007/ S10824-006-9026-3.

Yoo, B., \& Donthu, N. (2001). "Developing and validating a multidimensional consumerbased Brand equity scale". Journal of Business Research, 52(1), 1-14.

Zieba, M, \& O'Hagan, H. (2010). " Output Characteristics and Other Determinants of Theatre Attendance: An Econometric Analysis of German Data", Applied Economics Quarterly, 56(2), 147-174. 


\section{Anexo I. Composición del panel de expertos entrevistados}

\begin{tabular}{|c|c|c|}
\hline \multirow{6}{*}{ ARTISTAS ${ }^{1}$} & $\begin{array}{l}\text { José Soto Soto } \\
\text { José Mercé }\end{array}$ & $\begin{array}{l}\text { Premio Masters of Mediterranean Music del Mediterra- } \\
\text { nean Music Insitute (MMI) de Berklee College of Music. } \\
\text { Medalla de Andalucía } 2010.1 \text { Disco doble Platino, } 2 \\
\text { discos de Platino y } 2 \text { discos de oro. }\end{array}$ \\
\hline & $\begin{array}{l}\text { José Fernández } \\
\text { Torres } \\
\text { Tomatito }\end{array}$ & Medalla de Oro al Mérito en las Bellas Artes³ 2016. \\
\hline & $\begin{array}{l}\text { Marina Heredia Ríos } \\
\text { Marina Heredia }\end{array}$ & $\begin{array}{l}\text { Giraldillo }{ }^{4} \text { al cante 2016. Premio Mejor disco de Cante } \\
\text { Flamenco 2010, por la crítica Nacional de Flamenco. }\end{array}$ \\
\hline & $\begin{array}{l}\text { Francisco López- } \\
\text { Cepero García } \\
\text { Paco Cepero }\end{array}$ & Medalla de Oro al Mérito en las Bellas Artes 2003. \\
\hline & $\begin{array}{l}\text { Dra. Rocío Márquez } \\
\text { Limón } \\
\text { Rocío Márquez }\end{array}$ & $\begin{array}{l}\text { Doctora por la Universidad de Sevilla en Estudios Avan- } \\
\text { zados de Flamenco. Ganadora de la lámpara minera } \\
\text { en } 2008 \text { y del Giraldillo a la innovación } 2016 .\end{array}$ \\
\hline & $\begin{array}{l}\text { Juan Fernández } \\
\text { Montoya } \\
\text { Barullo }\end{array}$ & $\begin{array}{c}\text { Ganador del Concurso Nacional de Córdoba }{ }^{6} 2016 . \\
\text { Modalidad Baile. }\end{array}$ \\
\hline $\begin{array}{l}\text { POLÍTICO E } \\
\text { INVESTIGADOR }\end{array}$ & $\begin{array}{l}\text { Dr. Juan Manuel } \\
\text { Suárez Japón }\end{array}$ & $\begin{array}{c}\text { Consejero de Cultura y Medioambiente de la Junta } \\
\text { de Andalucía en el periodo 1990-1994. Catedrático } \\
\text { de Geografía Humana por la Universidad Pablo de } \\
\text { Olavide. }\end{array}$ \\
\hline \multirow[b]{2}{*}{$\begin{array}{l}\text { REPRESENTANTES } \\
\text { SGAE }^{7}\end{array}$} & $\begin{array}{l}\text { D. Javier Losada } \\
\text { Calvo }\end{array}$ & $\begin{array}{l}\text { Vicepresidente de Pequeño Derecho de SGAE. Miem- } \\
\text { bro del Consejo de Administración de AIE (1999-2012). }\end{array}$ \\
\hline & $\begin{array}{l}\text { D. José Manuel } \\
\text { Gamboa }\end{array}$ & $\begin{array}{c}\text { Periodista, escritor y productor musical español, } \\
\text { especializado en Flamenco. Actualmente desempeña } \\
\text { el cargo en SGAE de analista técnico musical en la } \\
\text { especialidad de Flamenco y es miembro del cuerpo } \\
\text { académico de la Cátedra de Flamencología de Jerez } \\
\text { de la Frontera. }\end{array}$ \\
\hline
\end{tabular}

1 Todos ellos son tanto intérpretes como compositores de buen número de las obras en las que participan.

2 Título honorífico creado por la Junta de Andalucía en 1985, que tiene por objeto reconocer "las acciones, servicios y méritos excepcionales o extraordinarios realizados en tiempos de paz por ciudadanos, grupos o Entidades andaluces, españoles o extranjeros" que representen "el ejercicio de virtudes individuales o colectivas que tengan como referencia la solidaridad y el trabajo en beneficio de los demás ciudadanos. Todo ello regulado por el Decreto 117/85, de 5 de junio.

3 Es una medalla que concede el Ministerio de Cultura de España a aquellas personas o instituciones que destaquen en los campos literario, dramático, musical, coreográfico, de interpretación, etc.

4 Reconocimiento que otorga la Bienal de Sevilla.

5 Reconocimiento que otorga el Festival Internacional del Cante de las Minas, celebrado cada año en el municipio de La Unión (en la Región de Murcia).

6 Reconocimiento otorgado por el Concurso Nacional de Arte Flamenco de Córdoba, certamen de carácter trianual que se celebra en la ciudad de Córdoba (España) desde 1956.

7 Sociedad General de Autores y Editores de España. Para más información: http://www.sgae.es/ es-ES/SitePages/index.aspx 


\begin{tabular}{|c|c|c|}
\hline \multirow{2}{*}{$\begin{array}{l}\text { REPRESENTANTES } \\
\text { AIE }^{8}\end{array}$} & $\begin{array}{l}\text { D. Álvaro } \\
\text { Hernández-Pizón }\end{array}$ & $\begin{array}{c}\text { Director de Asesoría jurídica y Estrategia de } \\
\text { Recaudación. }\end{array}$ \\
\hline & D. Pedro Rivas Prieto & Abogado de AIE. \\
\hline \multirow{3}{*}{$\begin{array}{l}\text { GESTORES } \\
\text { CULTURALES }\end{array}$} & $\begin{array}{l}\text { D. Cristóbal Ortega } \\
\text { Martos }\end{array}$ & Director artístico de la Bienal de Sevilla ${ }^{9}$ desde 2014. \\
\hline & $\begin{array}{l}\text { Dña. Isamay } \\
\text { Benavente Ferrera }\end{array}$ & Directora artística del Festival de Jerez de la Frontera ${ }^{10}$ \\
\hline & $\begin{array}{l}\text { D. Manuel Herrera } \\
\text { Rodas }\end{array}$ & $\begin{array}{c}\text { Actualmente Director artístico de los Jueves flamencos } \\
\text { organizados por la Fundación Cajasol }{ }^{11} \text { y miembro del } \\
\text { Consejo Asesor de la Bienal de Sevilla. Además fue } \\
\text { Director artístico de la Bienal de Sevilla en el periodo } \\
\text { 1997-2006. }\end{array}$ \\
\hline $\begin{array}{l}\text { CENTRO ANDALUZ DE } \\
\text { DOCUMENTACIÓN DEL } \\
\text { FLAMENCO }(\text { CADF })^{12}\end{array}$ & $\begin{array}{l}\text { Dra. Ana María } \\
\text { Tenorio Notario }\end{array}$ & $\begin{array}{l}\text { Responsable del Departamento de Documentación del } \\
\text { CADF }\end{array}$ \\
\hline
\end{tabular}

8 Sociedad de Artistas, Intérpretes o Ejecutantes de España. Para más información: https://www. aie.es/

9 La Bienal es el gran acontecimiento internacional del mundo del flamenco. Se celebra cada dos años en Sevilla, donde se dan cita los aficionados y profesionales del sector de todo el mundo. Para más información: http://www.labienal.com/

10 Jerez de la Frontera es considerado por los entendidos del Flamenco como uno de los puntos geográficos más importantes. La ciudad tiene un estilo propio y una idiosincrasia muy definida, siendo su Festival de uno de los más importantes ofrecidos por el mercado. Para más información: http://www.jerez.es/webs_municipales/festival_jerez/

11 Ciclo de conciertos y recitales de flamenco organizados por la Fundación Cajasol. Para más información: https://fundacioncajasol.com/tag/jueves-flamencos/

12 Se crea en 1987 para la conservación del arte jondo a través de una importante labor de recuperación, catalogación y difusión del patrimonio cultural andaluz. Para más información: http://www. juntadeandalucia.es/cultura/centroandaluzflamenco/ 


\section{Anexo II: Preguntas realizadas a los expertos entrevistados.}

a) En su opinión, ¿cuáles son los principales elementos que forman la obra flamenca? De los elementos mencionados, ¿cuáles son realizados por el intérprete?

b) En su concepto, ¿qué elementos reflejan la originalidad/creatividad de la contribución del intérprete a la obra flamenca?

c) ¿Cómo se puede medir dicha contribución?

d) Por favor, explique con el mayor grado de detalle la metodología que utiliza para su trabajo. Específicamente, ¿cómo participa en el proceso de creación artística? Describa el momento de la puesta en escena.

e) ¿Qué importancia tiene la intuición y la improvisación en el flamenco? 